\title{
THE IMPORTANCE OF INJECTION RATE IN ACHIEVING A SINGLE INTRAOCULAR GAS BUBBLE
}

\author{
G. WILLIAM AYLWARD and CHRISTOPHER J. LYONS \\ London
}

\begin{abstract}
SUMMARY
We investigated factors involved in achieving a single gas bubble following intravitreal gas injection during surgery for retinal detachment. A simple mathematical model of gas bubble dynamics within the eye was developed. The model indicates that the speed at which the bubble rises within the eye is proportional to the radius of the bubble. The flow rate required to maintain a single bubble increases as a power function of the bubble radius. In conclusion, the speed of injection is of paramount importance in the attainment of a single gas bubble.
\end{abstract}

Injection of gas into the vitreous cavity is often performed during external surgery for retinal detachment, in order to help drain subretinal fluid, reverse hypotony, or tamponade retinal breaks. ${ }^{1}$ The gas is usually injected using a syringe and a fine gauge needle inserted through the pars plana. It is important to obtain a single bubble of gas in order to maintain visualisation of the fundus. This is particularly important when employing the D-ACE sequence, ${ }^{2}$ in which gas injection precedes cryopexy and positioning of the explant. Unfortunately the creation of multiple small gas bubbles or 'fish eggs' is frequently reported after gas injection. This results in degradation of the ophthalmoscopic view, and can lead to subretinal gas bubbles. To avoid this complication previous authors have recommended that the needle be inserted through the highest point of the pars plana with the tip of the needle just visible. ${ }^{1}$ It has been our clinical impression, however, that the speed of injection is of paramount importance, though this is mentioned only rarely in the literature. $^{3}$ In this paper we consider the factors which may be important in the creation of a single bubble, and develop a simple mathematical model of gas bubble formation within the eye.

Correspondence to: Mr G. W. Aylward, Moorfields Eye Hospital, London EC1V 2PD, UK.

\section{MATERIALS AND METHODS}

Consider a spherical gas bubble of radius $r$ forming around the point of a needle through which gas is being injected into a liquid of density $\rho$ and viscosity $\mu$. The bubble will remain single as long as the needle tip remains within it as gas is being injected. This will depend on the motion of the bubble relative to the needle tip. If the bubble is in contact with the highest point of the eye, then it will not move and it will remain single as further gas is injected. However, until this position is reached it will move according to the balance of forces upon it. The bubble is buoyant in the liquid and will tend to rise due to an upward buoyancy force. By the principle of Archimedes this is equal to the weight of liquid displaced by the bubble, given by:

$$
\text { Upward force }=\frac{4}{3} \pi r^{3} \rho g
$$

where $g$ is Newton's constant. As the bubble moves through the liquid it will experience a retarding force, $D$, due to drag. Forces on the bubble due to surface tension are ignored in this model. The drag force can be calculated using principles of fluid mechanics. ${ }^{4}$ In fact, vitreous exhibits non-Newtonian behaviour, but variation in its viscosity is small, ${ }^{5}$ so that for the purposes of this mathematical model vitreous can be considered to be Newtonian. For small spheres in very viscous media, the drag is given by Stokes' law:

$$
D=6 \pi r \mu u
$$

where $u$ is the velocity of the bubble. However, this relationship does not apply when the ratio of inertial forces to viscous forces is high. This ratio is known as the Reynolds number, $R e$, and is given by:

$$
R e=2 r u \rho / \mu
$$

Stokes' law is found by experiment to be invalid for Reynolds numbers above 0.1 , which is unfortunately 
the case for our model. Instead, the total drag force is given by:

$$
\text { Total drag }=C_{\mathrm{D}} \frac{1}{2} \rho u^{2} A
$$

where $C_{\mathrm{D}}$ is the drag coefficient and $A$ is the frontal area of the body in motion (in this case a sphere, so that $\left.A=\pi r^{2}\right) .{ }^{4} C_{\mathrm{D}}$ varies with the size of the sphere and the velocity, and has been determined experimentally for a range of Reynolds numbers. In the steady state the buoyancy is balanced by the total drag, so that:

$$
C_{\mathrm{D}} \frac{1}{2} \rho u^{2} \pi r^{2}=\frac{4}{3} \pi r^{3} \rho g
$$

ignoring the contribution of the weight of the gas bubble, which is small compared wth its buoyancy. Solving this equation for the velocity, $u$, is not straightforward since $C_{\mathrm{D}}$ is a function of $u$. However, it can be rearranged in terms of $C_{\mathrm{D}}$ and $R e$ :

$$
\left\{\frac{3}{4} \mathrm{C}_{\mathrm{D}}(R e)^{2}\right\}^{\frac{1}{3}}=2 r\left\{g \rho^{2} / \mu^{2}\right\}^{\frac{1}{3}}
$$

The left-hand side of the equation can be related to the Reynolds number by reference to standard graphs for drag relations of isolated smooth spheres, ${ }^{4}$ giving a value for $R e$, and hence $u$, from equation (3). The relationship between the quantity in equation (6) and the Reynolds number is approximately linear for Reynolds numbers in the range 0.1 to 105 , and can therefore be expressed by the equation of a straight line $(y=a x+b)$ thus:

$$
\log _{10} R e=2 \log _{10} X-1
$$

where $X$ is the left-hand side of equation (6). Combining equations (3), (6) and (7) gives an expression for the velocity of a gas bubble in terms of its radius and the viscosity of the liquid. It is more convenient to use the kinematic viscosity, $v=\mu / \rho$ than $\mu$ :

$$
u=\frac{v}{2 r} 10^{\left\{2 \operatorname { l o g } _ { 1 0 } \left(2 r \sqrt[3]{\left.\left.g / v^{2}\right)-1\right\}}\right.\right.}
$$

This simplifies to:

$$
u=\frac{1}{5} \sqrt[3]{g^{2} / v r}
$$

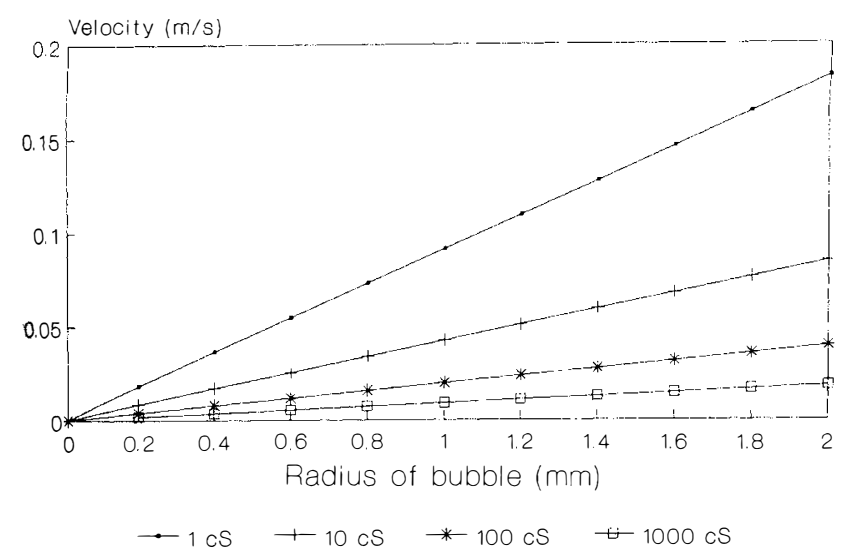

Fig. 1. The upward velocity of gas bubbles of various radii in liquids of various viscosities.
Hence as the bubble increases in size, it also rises faster. If the bubble has not reached the highest point in the eye and is still in motion, it will leave the tip of the needle if its velocity exceeds the rate at which its radius is increasing. The rate of increase in radius is a function of the rate at which the gas is being injected. Hence the critical flow rate, $f$, at which the bubble would leave the tip of the needle is given by:

$$
f=\frac{4}{3} \pi u^{3}
$$

assuming the bubble to be spherical. Therefore, for a constant flow rate of $f$, the size of the gas bubble as it leaves the tip of the needle is given by substituting equation (9) for $u$ in equation (10):

$$
f=\frac{4}{375} \pi g^{2} r^{3} / v
$$

Suppose the needle tip is inserted a distance $x$ from the highest point of the eye. In order to achieve a single bubble, the flow rate must be sufficient to create a bubble of radius $x / 2$, otherwise it will leave the tip of the needle before its motion is arrested. Hence the flow rate required is:

$$
f=\frac{1}{750} \pi g^{2} x^{3} / v
$$

An experimental chamber was constructed from clear polymethylmethacrylate in order to illustrate some of the principles developed in the mathematical model. A cylindrical chamber with straight ends was immersed in a clear bath containing glycerol. Air was injected at various flow rates through small holes in the roof of the chamber. The bubbles that were produced were then photographed.

\section{RESULTS}

The relationship between upward velocity and bubble size described by equation (9) is illustrated graphically in Fig. 1. The upward velocity for a range of bubble sizes and liquid viscocities is shown. The viscosity of vitreous varies, but typical values are of the order of $1-2 \mathrm{cSt}^{5}$ The viscosity of water is $1 \mathrm{cSt}$ at $20.2{ }^{\circ} \mathrm{C} .{ }^{4}$ The relationship between the critical flow rate, $f$, and the insertion distance of the needle tip,

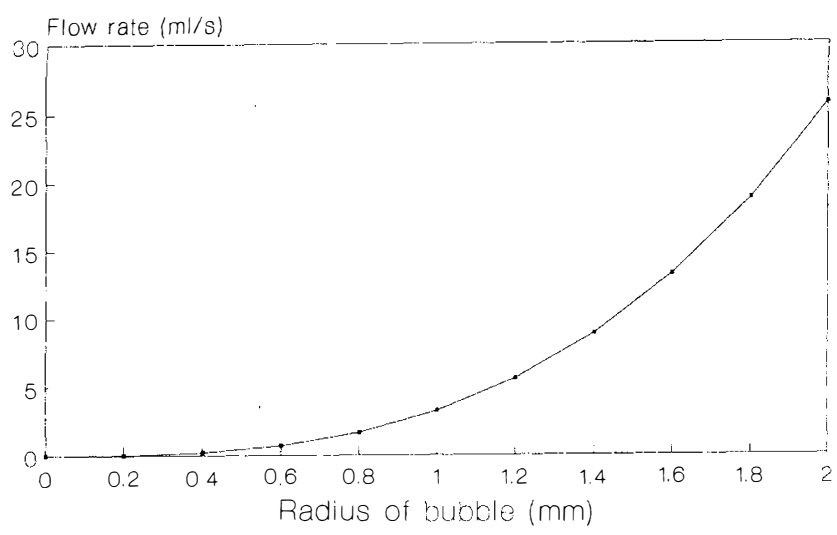

Fig. 2. The way in which the flow rate required for a single bubble varies with the radius of the bubble. 


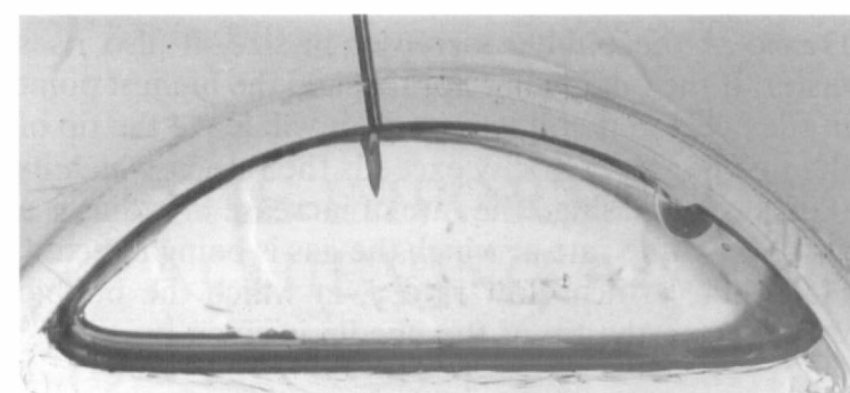

Fig. 3. Gas being injected with the tip of the needle just within the cavity at the highest point. A single bubble is easily achieved.

described in equation (12), is shown graphically in Fig. 2. It can be seen that the critical flow rate increases as a cubic power function of the insertion distance $x$. For example, to prevent a bubble of $1 \mathrm{~mm}$ radius leaving the tip of the needle requires a flow rate of $4 \mathrm{ml} / \mathrm{s}$. Once the bubble has increased in size to $2 \mathrm{~mm}$ radius, however, an injection rate of $25 \mathrm{ml} / \mathrm{s}$ is required. The creation of a single bubble is therefore relatively easy if the needle is positioned so that the tip is near the highest point of the eye (Fig. 3). If the needle is not in an ideal position and the flow rate is slow, multiple bubbles are the inevitable result (Fig. 4). However, a single bubble can be achieved with the needle in the same position if the flow rate is sufficiently rapid (Fig. 5).

\section{DISCUSSION}

This model of bubble creation shows the importance of flow rate in achieving a single bubble - a factor which has not been emphasized previously. Recommendations currently in the literature include inserting the needle at the highest point in the globe, and withdrawing the needle so that the tip is just visible. ${ }^{1}$

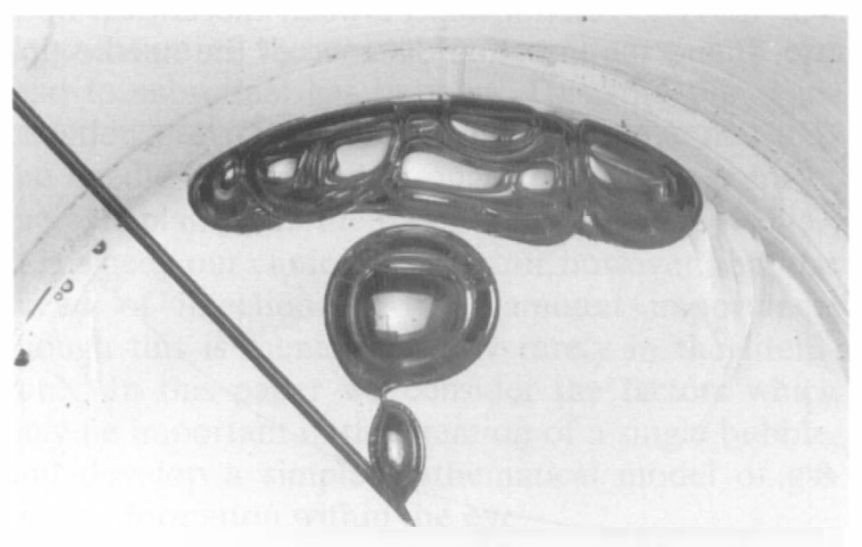

Fig. 4. Gas being injected slowly with the needle inserted some way into the eye, and not at the highest point. Multiple bubbles are the result.

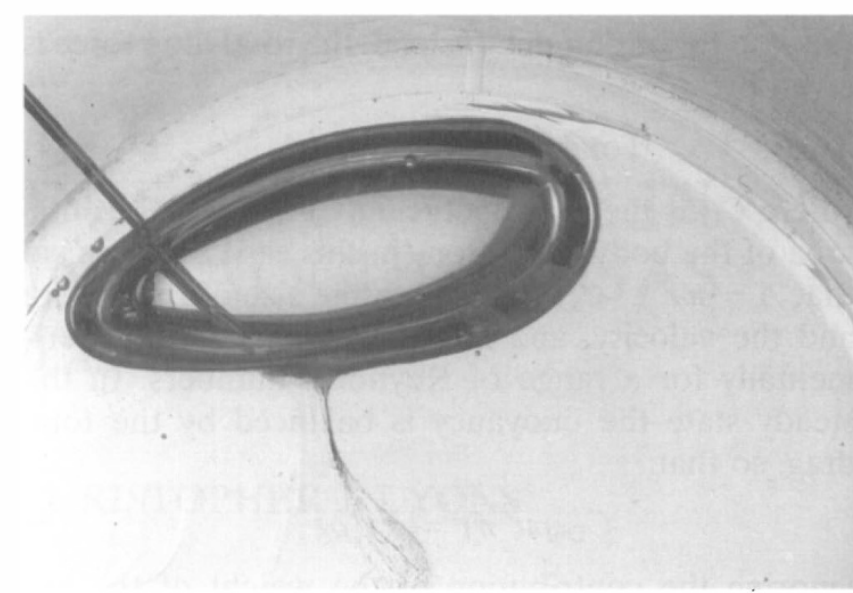

Fig. 5. Gas being injected with the needle in a similar position as in Fig. 4 but at a higher flow rate. A single bubble is achieved.

The needle is inserted through the pars plana, but the limits of ophthalmoscopy are such that there will be a finite distance, say $1 \mathrm{~mm}$, between the tip of the needle and the pars plana epithelium. It is inadvisable to withdraw the tip from view because of the risk of injecting air into the supra-choroidal space. Are these conditions sufficient to achieve a single bubble? Our model suggests not. Fig. 2 indicates that multiple bubbles would result if the injection rate is less than $4 \mathrm{ml} / \mathrm{s}$. The speed of injection is therefore a vital factor in obtaining a single bubble, even if the other conditions are met. Equation (12) shows that a single bubble can be achieved with the needle tip inserted to any distance, but because of the cubic relationship, the flow rate becomes impractically large for distances greater than a few millimetres (Fig. 2).

In conclusion, our model confirms the importance of needle tip position in avoiding 'fish eggs', but indicates that the rate of injection is equally important.

This work was supported by a grant from the Special Trustees of Moorfields Eye Hospital

Key words: Retinal detachment surgery, Intraocular gas injection, Insoluble gases, Viscosity, Vitreous.

\section{REFERENCES}

1. Norton EWD. Intraocular gas in the management of selected retinal detachments. Ophthalmology 1973; 77:85-98.

2. Gilbert C, McLeod D. D-ACE sequence for selected bullous retinal detachments. Br J Ophthalmol 1985; 69:733-6.

3. Hilton GF, Grizzard WS. Pneumatic retinopexy: a two step outpatient operation without conjunctival incision. Ophthalmology 1986;93:626-41.

4. Massey BS. Mechanics of fluids, 6th edn. London: Chapman and Hall, 1989.

5. Kawano S, Honda Y, Negi A. Effects of biological stimuli on the viscosity of the vitreous. Acta Ophthalmol (Copenh) 1982;60:977-91. 